\title{
Phase organization of mesogen-decorated spherical nanoparticles
}

\section{SILVIA ORLANDI* AND CLAUDIO ZANNONI}

Dipartimento di Chimica Industriale "Toso Montanari”, Università di Bologna, Viale Risorgimento 4, 40136 Bologna, Italy

We present a Monte Carlo simulation study of the phase behaviour and molecular organizations observed in a system of nanoparticles constituted by a central spherical core with attached mesogenic groups, which we model with a set of suitable Gay-Berne units connected by flexible spacers. We show that the choice of radial or tangential orientation of the attached mesogenic groups with respect to the surface of the core is crucial for the resulting phase behavior.

Keywords: Computer simulation, Monte Carlo, mesogen-coated nanoparticles

\section{Introduction}

A necessary requirement for the formation of liquid crystal (LC) phases has long been considered the anisotropy of the constituent particles, be it rodlike, calamitic or flatlike, discotic [1]. The situation has recently changed with the discovery of a variety of liquid crystals with shape deviating from these canonical ones like bent, "banana" [2,3], phasmidic [4], dendrimeric [5], fullerenic [6-9] mesogens.

A particularly striking case is that of spherical nanoparticles (NP), typically gold ones, decorated with mesogens attached radially or

* Address correspondence to Silvia Orlandi, Dipartimento di Chimica Industriale "Toso Montanari", Università di Bologna, Italy. Fax:+39-0512093690; E-mail:s.orlandi@unibo.it 


\section{MESOGEN-DECORATED NANOPARTICLES: A MC STUDY}

tangentially with a flexible thiol terminated spacer group to the gold surface [10-15], similar in various ways to first generation dendrimers, and forming various liquid crystalline phases.

While simulations at space resolutions going from the mesoscopic scale, with positions arbitrarily fixed on lattices [16], to the molecular scale with off-lattice coarse grained [17] or even down to atomistic [18] level of detail exist for many mesogenic systems of various complexity [1923], we are not aware of any modelling and simulation studies trying to understand the phases and the ordering process in these exotic systems which, on the other hand, have many promising applications, e.g. in the field of optical metamaterials and more generally of novel functional materials with hybrid properties [24-27].

In this work we intend to put forward a first simple model to treat spherical nanoparticles decorated on the surface with mesogens and study their phase behavior.

\section{Model and Simulation}

We set out to implement the essential characteristic of two classes of nanoparticles architectures, where the mesogens are attached to the central unit either at an end, i.e. radially, or at a side, i.e. tangentially, (see Fig. 1).

Each model consists of one central sphere representing the nanoparticle core $(\mathrm{N})$ and eight equivalent uniaxial Gay-Berne elongated ellipsoids representing the substituent mesogens (M). We assume that the spherical core site is connected to the mesogens through a flexible spring, with the bonding sites placed on surface of sphere and ellipsoids; bonds can only stretch and bend but not break during the simulation [28]. 

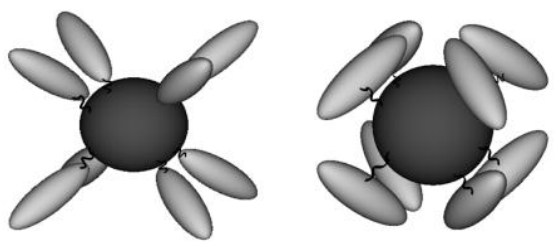

\begin{tabular}{|c|c|c|c|c|c|c|}
\hline Site & $\sigma_{s}$ & $\sigma_{e}$ & $\sigma_{c}$ & $\varepsilon_{s}$ & $\varepsilon_{e}$ & $n_{\text {bnd }}$ \\
\hline $\mathrm{N}$ & 3.0 & 3.0 & 3.0 & 0.5 & 0.5 & 8 \\
\hline $\mathrm{M}$ & 1.0 & 3.0 & 1.0 & 1.0 & 0.2 & 1 \\
\hline
\end{tabular}

Figure 1. Schematic drawing of the radially (left) and laterally (right) grafted model nanoparticles. Dimensions and interaction parameters of the nanoparticle $(\mathrm{N}$, dark gray) and mesogenic (M, light gray) interaction sites, expressed in terms of the parameters $\sigma_{0}, \varepsilon_{0}$ that serve to scale the simulation energy and distance units are reported in the Table.

The corresponding bonded interactions are expressed with harmonic contributions depending on the separation $s_{i j}=\left|\mathbf{p}_{j}-\mathbf{p}_{i}\right|$, and the bending angle $\theta_{i j}=\cos ^{-1}\left(\mathbf{u}_{i} \cdot \mathbf{u}_{j}\right)$ between two linked bonding sites:

$$
U^{B N D}=k_{s}\left(s_{i j}-s_{e q}\right)^{2}+k_{\theta}\left(\theta_{i j}-\theta_{e q}\right)^{2}
$$

where $\mathbf{p}$ and $\mathbf{u}$ are the positions and the orientations of the bonding sites, $s_{e q}$ and $\theta_{e q}$ the equilibrium spacer length and bend angle, and $k_{s}, k_{\theta}$ the stiffness parameters of the spacer. For both the cases we have used the same spacer, with bending harmonic constant $k_{\theta}=100 \mathrm{rad}^{-2}$, bond angle $\theta_{e q}=180^{\circ}$, stretching harmonic constant $k_{s}=200 \quad \sigma_{0}{ }^{-2}$ and equilibrium length $s_{e q}=0.5 \sigma_{0}$.

Each of the sites on a nanoparticle, spherical $(\mathrm{N})$ and mesogenic $(\mathrm{M})$, interacts via a generalized GB repulsive-attractive site-site potential $[17,29,30]$ and the potential between a pair of nine-sites molecules is the sum of all possible interactions between the sites of two molecules, namely: 


$$
U^{G B} \equiv U_{M M}^{G B}+U_{N N}^{G B}+U_{M N}^{G B}
$$

The interaction potential is a special case of the expression developed for dissimilar biaxial particles $A, B[31]$ :

$$
\begin{gathered}
U_{A B}^{G B}\left(\omega_{A}, \omega_{B}, \hat{\mathbf{r}}_{A B}\right)=4 \varepsilon_{0} \varepsilon_{A B}^{v}\left(\omega_{A}, \omega_{B}, \hat{\mathbf{r}}_{A B}\right) \varepsilon_{A B}^{\prime \mu}\left(\omega_{A}, \omega_{B}, \hat{\mathbf{r}}_{A B}\right) \times \\
\left\{\left[\frac{\sigma_{c}}{r_{A B}-\sigma_{A B}\left(\omega_{A}, \omega_{B}, \hat{\mathbf{r}}_{A B}\right)+\sigma_{c}}\right]^{12}-\left[\frac{\sigma_{c}}{r_{A B}-\sigma_{A B}\left(\omega_{A}, \omega_{B}, \hat{\mathbf{r}}_{A B}\right)+\sigma_{c}}\right]^{6}\right\}
\end{gathered}
$$

where $\omega_{A}$ and $\omega_{B}$ are the molecular orientations in terms of Euler angles [29], $\mathbf{r}_{A B} \equiv \mathbf{r}_{A}-\mathbf{r}_{B}$ is the site-site vector, $\sigma_{A B}\left(\omega_{A}, \omega_{B}, \hat{\mathbf{r}}_{A B}\right)$ anisotropic distance function and $\sigma_{c}$ the minimum contact distance, $\varepsilon_{A B}^{v}\left(\omega_{A}, \omega_{B}, \hat{\mathbf{r}}_{A B}\right)$ is the interaction strength at zero separation and $\varepsilon^{\prime \mu}{ }_{A B}\left(\omega_{A}, \omega_{B}, \hat{\mathbf{r}}_{A B}\right)$ the ad-hoc interaction term as defined in [31], containing the empirical exponents $\mu$ and $v$, used for tuning the potential angular dependence, that we select respectively equal to 1 and 3 .

This expression is a generalized version of the GB potential, that can be used for a straightforward calculation of the mixed interaction potential between dissimilar particles $A, B$ by simply employing $\sigma_{c}=\frac{1}{2}\left[\sigma_{c}^{A}+\sigma_{c}^{B}\right]$. Actually the GB potential depends only on the ratios of the sigmas and the epsilons that we choose as $\sigma_{e} / \sigma_{s}=1$ and $\varepsilon_{s} / \varepsilon_{e}=1$ for the $\mathrm{N}$ (spherical) site and $\sigma_{e} / \sigma_{s}=3$ and $\varepsilon_{s} / \varepsilon_{e}=5$ and for the $\mathrm{M}$ (rod-like) site (see Fig. 1) where $s$ and $e$ stand for side-to-side and end-to-end dimer arrangements. The parameterization for the $M$ sites in case of systems of uniaxial ellipsoid particles gives a broad temperature range of nematic, smectic and crystal phases [17,28,30,32]. 


\section{MESOGEN-DECORATED NANOPARTICLES: A MC STUDY}

We performed a large number of Monte Carlo (MC) experiments in the isobaric-isothermal $(N P T)$ ensemble, at adimensional pressure $P^{*} \equiv$ $P \sigma_{0}{ }^{3} / \varepsilon_{0}=5$ and temperature $T^{*} \equiv k_{B} T / \varepsilon_{0}$, on samples of $N=125$ decorated nanoparticles (i.e. 125 spherical sites and 1000 rod sites).

For both radial or tangential attachment types, simulations started from well equilibrated configurations in the isotropic phase. The simulations were run in a cooling-down sequence of Monte Carlo runs, each of them starting from the final equilibrated configuration obtained at the previous temperature. The length of equilibration MC runs varied starting from 0.5 millions cycles, for the first isotropic configurations, to 1.0 million cycles on the approach of ordered phases at lower temperatures, each cycle corresponding to $N$ attempted single-particle MC moves and the acceptance ratio being 0.4 . From a practical point of view we have used an orthogonal simulation box with $3 \mathrm{D}$ periodic boundary conditions, allowing its sides to fluctuate independently during the MC evolution so as to let the system adjust to its equilibrium density at the given thermodynamic state and to help avoiding formation of cavities in the sample.

During production run thermodynamic observables have been accumulated for averaging and data analysis every 20 cycles.

The average values of dimensionless energy $\left\langle U^{*}\right\rangle$ and order parameter $\left\langle P_{2}\right\rangle=\left\langle\frac{3}{2}\left(\hat{\mathbf{z}}_{i} \cdot \hat{\mathbf{Z}}\right)^{2}-\frac{1}{2}\right\rangle$, showing the average orientational order of the molecular $\hat{\mathbf{z}}_{i}$ axis with respect to the director orientation defining unit vector $\hat{\mathbf{Z}}$, were determined from the simulations.

Characterization of the phase was accomplished first by calculating density correlation functions along the director (along $\mathrm{Z}$ axis) and 
perpendicular to it (XY plane rod-rod and sphere-sphere correlation functions) for both $\mathrm{M}$ and $\mathrm{N}$ sites:

$$
\begin{aligned}
& g_{\|}(r)=\frac{1}{\pi R_{\|}^{2} \rho}\left\langle\delta\left|\left(\mathbf{r}-\mathbf{r}_{i j}\right) \cdot \mathbf{Z}\right|\right\rangle_{i j} \equiv \frac{1}{\pi R_{\|}^{2} \rho}\left\langle\delta\left(z-z_{i j}\right)\right\rangle_{i j} \\
& g_{\perp}(r)=\frac{1}{\pi R_{\perp}^{2} \rho}\left\langle\delta\left|\left(\mathbf{r}-\mathbf{r}_{i j}\right)\right| \times \mathbf{Z}\right\rangle_{i j}
\end{aligned}
$$

here $z_{i j}=r_{i j} \cos \beta_{r_{i j}}$ is measured with respect to the principal $Z$ axis of the director frame, i.e. $\beta_{r_{i j}}$ is the angle formed between intermolecular vector $\mathbf{r}_{i j}$ and the director $\mathbf{Z}, R_{\|}$is the radius of the corresponding cylindrical sampling regions, whereas $R_{\perp M}$ is the radius of the rod-like ellipsoid $R_{\perp, D}$ is the radius of the sphere, $\cdot$ and $\times$ stand for the scalar and vector products respectively, and the average $\langle\ldots\rangle_{i j}$ is computed over all molecular pairs. The density correlation function $g_{\|}(r)$ is particularly useful for the identification of the layered structures present in the systems, while the $g_{\perp}(r)$ is helpful for investigations of the order inside the layers and gives the information about the positional order of spherical and rod sites, respectively.

\section{Results}

Temperature dependence profiles of the main observables of the two studied systems, with radial and tangential substitution, i.e. density and orientational order parameter $\left\langle P_{2}\right\rangle$, at pressure $P^{*}=5$, are reported in Figs. 2 and 5. Chosen snapshots presenting molecular assemblies obtained in simulations are given in Figs. 3 and 6, while correlation functions in Figs. 4 and 7. 

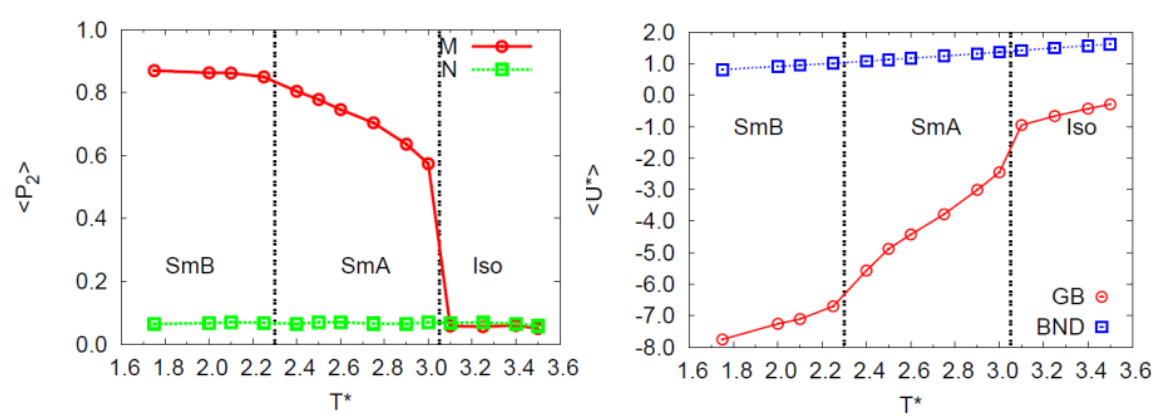

Figure 2. Orientational order parameter $\left\langle P_{2}\right\rangle$ and average energy $\left\langle U^{*}\right\rangle$ per decorated nanoparticle with radial substitution, as a function of $T^{*}$, at dimensionless pressure $P^{*}=5$.

Simulations show that, upon decreasing temperature, the system with radial substitution undergoes phase changes accompanied by an increase of orientational and translational order with Isotropic, Smectic A and Smectic B phases. Quite interestingly, even in the range of temperatures 3.0-2.7, characterized by orientational order parameter typical of nematic phases, we observe a strong structuration in layers for both $\mathrm{M}$ and $\mathrm{N}$ sites, as indicated by the density correlation functions along the director $g_{\|}^{N N}(r)$ and $g_{\|}^{M M}(r)$ (see Fig. 4).

Both functions show a sharp modulation, suggesting that spheres and rods are arranged in sublayers; maxima are separated by the distance $r^{*} \approx 5.0$ (roughly half of decorated nanoparticle in the linear conformation) that implies a fully interdigitated structure of the mesogenic layers.

The existence of this phase organization is supported by the snapshots of equilibrated configurations reported in Fig. 3: at the lowest temperatures the mesogenic groups tend to align with each other and to segregate at the two ends of the spherical nanoparticle, enhancing phase separation, 
as a microsegregation of both $\mathrm{M}$ and $\mathrm{N}$ sites in continuous quasi planar sub-layers is observed.
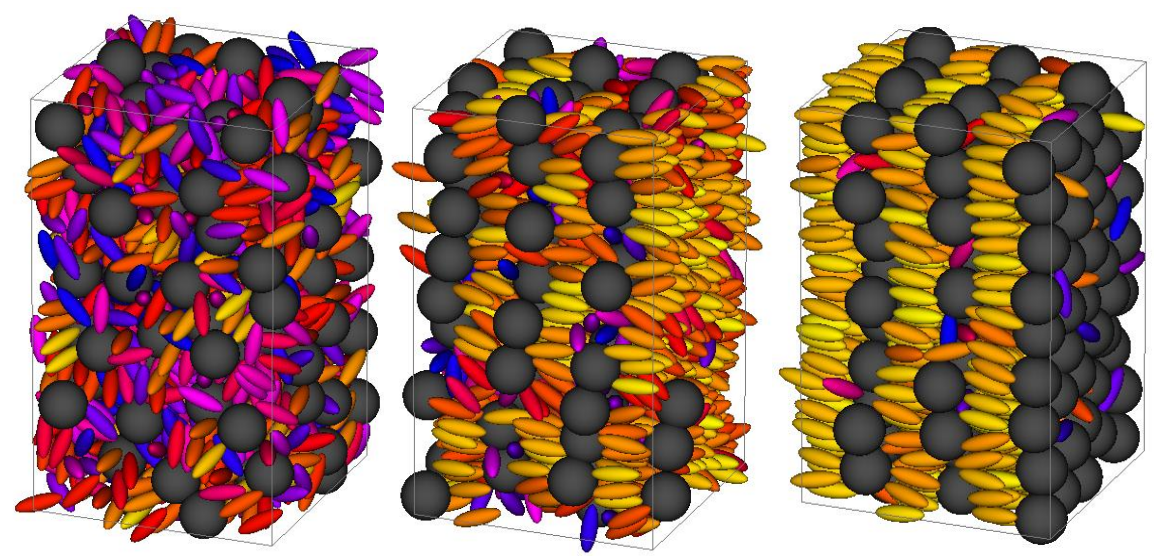

Figure 3. Snapshots of samples of 125 radially grafted nanoparticles at $T^{*}=3.2$ (Nematic), 3.0 (Smectic A), 2.0 (Smectic B). Dimensionless pressure is $P^{*}=5$. The spherical central nanoparticle is represented by a dark gray sphere, while mesogens are color coded according to their orientation.

From the correlation functions in the direction perpendicular to the director in the sublayer made of cores, $g_{\perp}{ }^{N N}$, and in the sublayer made of mesogens, $g_{\perp}{ }^{M M}$, it can be noticed (see Fig. 4) that the rod-like units exhibit higher positional order than the spherical ones. In particular, at $T^{*}=2.0$ it is evident the characteristic splitting of the second peak around $r^{*} \cong 2.0$, indicating an hexatic order typical of Smectic B phases, while, at the intermediate temperature $T^{*}=3.0$, the rapid decay of the density correlation, and the lack of double peaks, highlight the liquidlike order inside the layer, characteristic of Smectic A phases. 

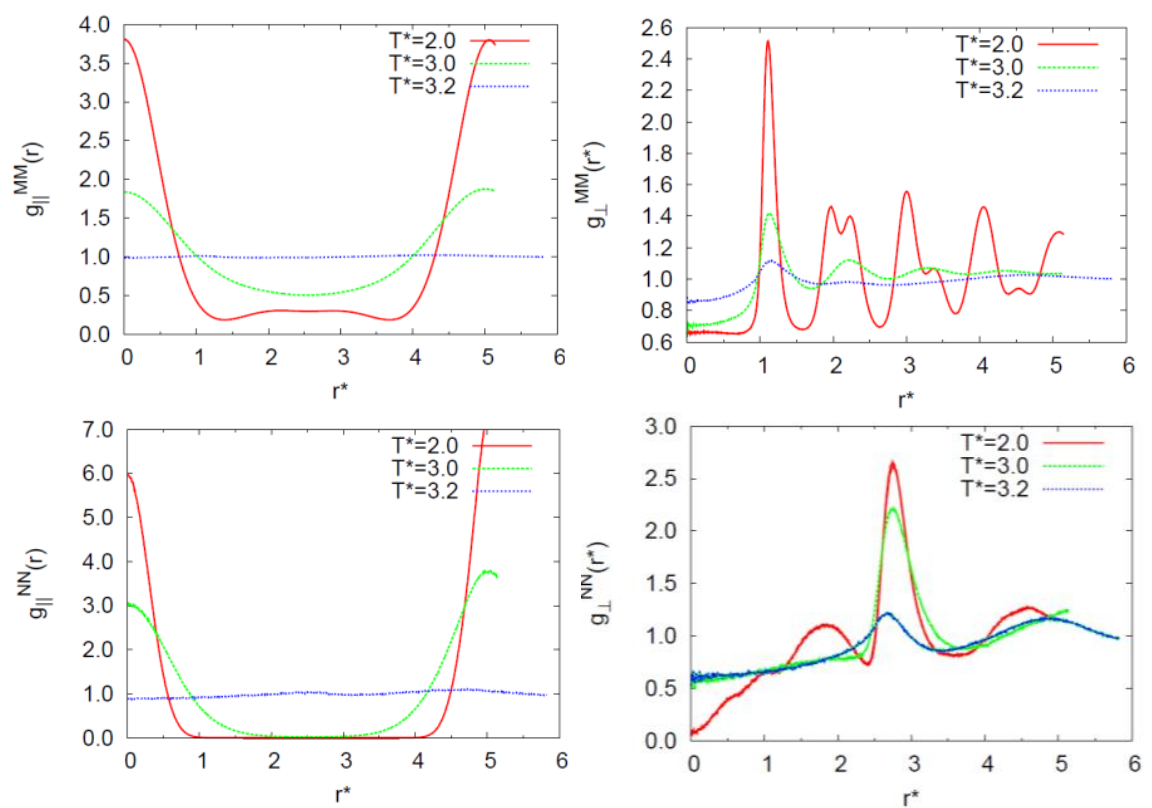

Figure 4. Density correlation function along the director and perpendicular to it, for the system with radial substitution at three temperatures corresponding to Isotropic $\left(T^{*}=3.2\right.$, dashed line), Smectic A $\left(T^{*}=3.0\right.$, dotted lines), Smectic B $\left(T^{*}=3.2\right.$, solid line $)$ phases.

Turning to the model with tangentially-attached mesogenic groups, our simulations show that the system yields mesomorphism with isotropic and Smectic A phases. Comparing with the radially-attached model we observe a general reduction of the liquid crystalline orientational order parameter $\left\langle P_{2}\right\rangle_{M}$ and a shift to lower temperatures of the IsotropicSmectic A transition (Fig. 5).

The density correlation functions along the director for the mesogenic sites, $g_{\|}{ }^{M M}(r)$, shows, in smectic phase, a modulation with maxima separated by the distance $r^{*} \approx 2.7$; instead, and differently from the 


\section{MESOGEN-DECORATED NANOPARTICLES: A MC STUDY}
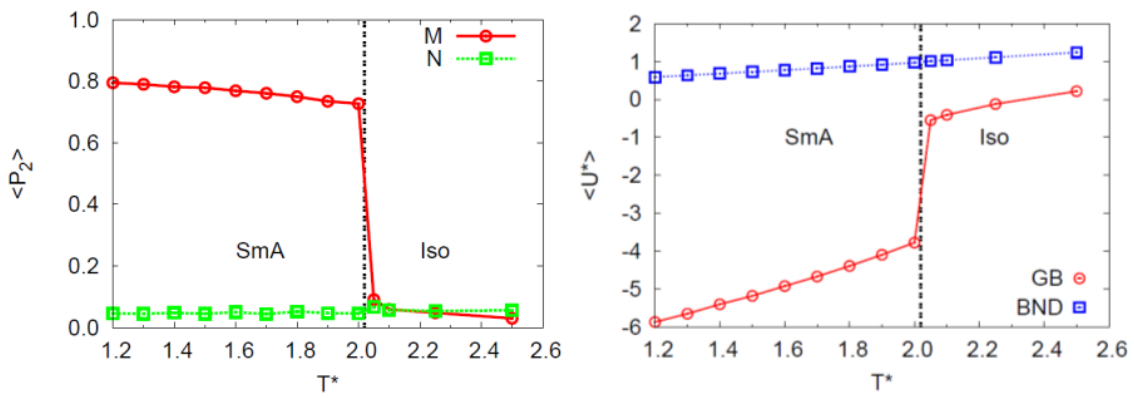

Figure 5. Orientational order parameter $\left\langle P_{2}\right\rangle$ and average energy $\left\langle U^{*}\right\rangle$ per decorated nanoparticle with tangential substitution, as a function of $T^{*}$, at dimensionless pressure $P^{*}=5$.

radially substitution case, the $g_{\|}^{N N}(r)$ shows a more complicated structure of peaks with maxima still separated by $r^{*} \approx 2.7$.
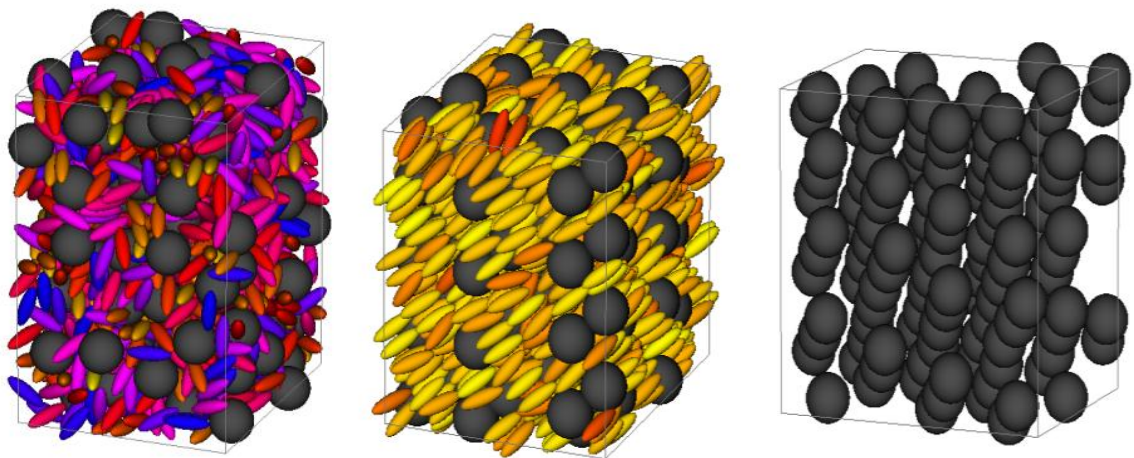

Figure 6. Snapshots of molecular organizations of the 125 laterally grafted nanoparticles sample at $T^{*}=2.2$ (left), and 1.8 (center). The spherical central nanoparticle is represented by a dark gray sphere, while mesogens are color coded according to their orientation. To help in the visualization of the columnar stacks, only the spherical sites have been represented and a top view has been chosen for the snapshot on right, relative to temperature $T^{*}=1.8$. 
Correspondingly, the snapshots of typical molecular organizations obtained from simulations, even at the lowest temperature (see Fig. 6), evidence that the central spherical cores no longer organize in continuous planes, but rather are packed in columnar stacks separated by a distance $r^{*} \approx 4.0$ and regularly arranged in the whole box volume. On the other hand, mesogenic sites still tend to organize in layers, even if discontinuous.
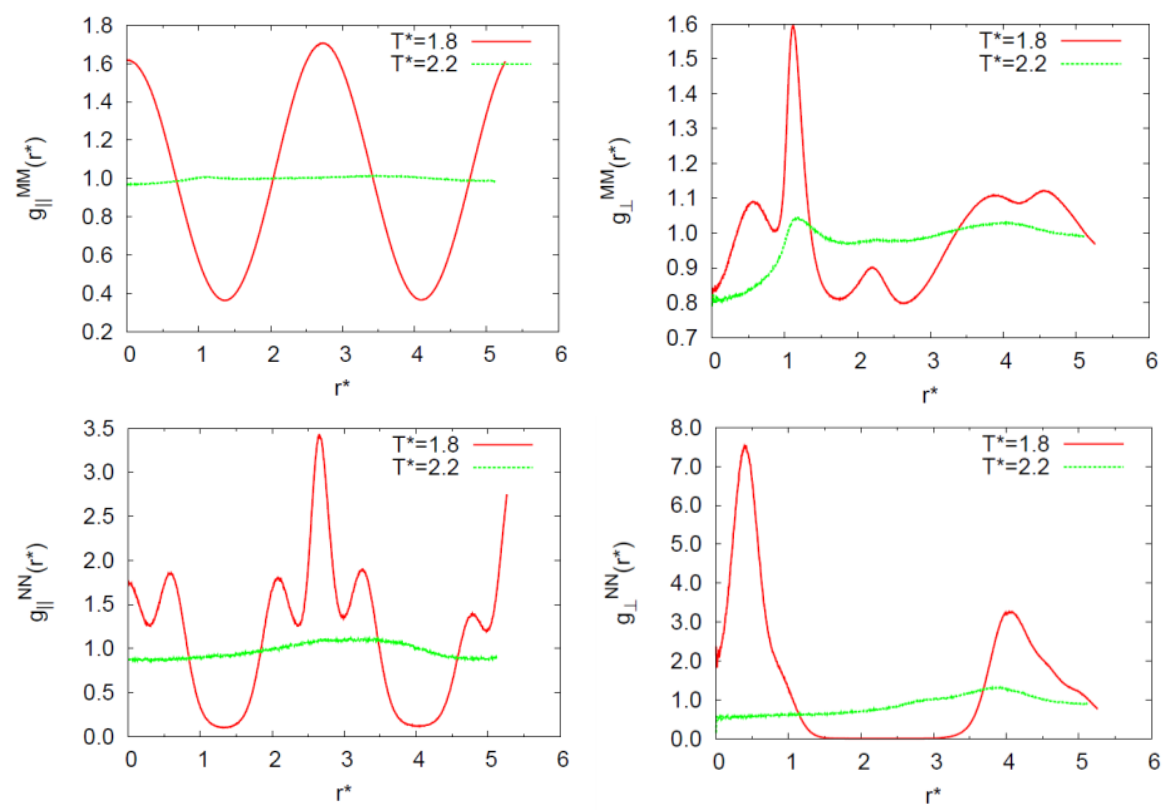

Figure 7. Density correlation function along the director and perpendicular to it for the system of nanoparticles with tangential substitution in Isotropic $\left(T^{*}=2.2\right.$, dashed line $)$ and in Smectic $\mathrm{A}\left(T^{*}=1.8\right.$, solid line) phases.

Trying to rationalize this molecular arrangement, we observe that for the tangential substitution, the bonded mesogens cannot migrate at the two ends of the spherical nanoparticle, and remain distributed over 
its surface; thus the linear conformation, and consequently the spheres segregation are prevented.

\section{Conclusions}

We have studied, by means of Monte Carlo simulations, the molecular organizations for two classes of nanoparticle architectures with mesogens attached to the central spherical core in a radial or in a tangential way.

The simulations show that upon lowering temperature the system with radial substitution yields microsegregation of both spherical and mesogenic sites with consequent formation of quasi-planar sublayers; the system with lateral substitution gives a very different aggregation patterns with spherical sites segregate both into regularly packed columnar stacks and in (discontinuous) layer. The internal flexibility appears essential for this peculiar self-assembling feature. Even though this is a very simple model for mesogen decorated nanoparticles, and thus a detailed comparison with experiment would probably be inappropriate at this stage, since no attempt has been made to tune nanoparticle size, spacer length and number of mesogenic ligands, it is comforting to see that the striking observation of liquid crystal phases formation for these unconventional shaped particles is reproduced and that the organization found is quite similar to what has been assumed in experimental work [10-16,24].

\section{Acknowledgments}

We thank MIUR PRIN national project "Novel ordered systems for high response molecular devices" (2009N5JH4F) for financial support. 


\section{MESOGEN-DECORATED NANOPARTICLES: A MC STUDY}

\section{References}

[1] Blinov, L. (2011). Structure and Properties of Liquid Crystals, Springer, Dordrecht.

[2] Link, D. R., Natale G., Shao, R., Maclennan, J. E., Clark, N. A., Korblova E., \& Walba, D. M. (1997). Spontaneous Formation of Macroscopic Chiral Domains in a Fluid Smectic Phase of Achiral Molecules, Science, 278, 19241927.

[3] Francescangeli, O., Stanic, V., Torgova, S. I., Strigazzi, A., Scaramuzza, N. Ferrero, C., Dolbnya, I. P., Weiss, T. M., Berardi, R. et al. (2009). Ferroelectric Response, \& Induced Biaxiality in the Nematic Phase of a Bent-core Mesogen, Adv. Funct. Mater., 19, 2592-2600.

[4] Yoon, S. J., Kim, J. H., Kim, K. S., Chung, J. W., Heinrich, B., Mathevet, F., Kim, P., Donnio, B., Attias A. J. et al. (2012). Mesomorphic Organization and Thermochromic Luminescence of Dicyanodistyrylbenzene-based Phasmidic Molecular Disks: Uniaxially Aligned Hexagonal Columnar Liquid Crystals at Room Temperature with Enhanced Fluorescence Emission and Semiconductivity, Adv. Funct. Mater., 22, 61-69.

[5] Donnio, B., Buathong, S., Bury, I., \& Guillon, D. (2007). Liquid Crystalline Dendrimers, Chem. Soc. Rev., 36, 1495-1513.

[6] Deschenaux, R., Donnio, B., \& Guillon, D. (2005). Liquid-crystalline Fullerodendrimers, New J. Chem., 31, 1064-1073.

[7] Bushby, R. J., Hamley, I. W., Liu, Q. Y., Lozman, O. R., \& Lydon, J. E. (2005). Self-assembled Columns of Fullerene, J. Mat. Chem., 15, 4429-4434.

[8] Sawamura, M., Kawai, K., Matsuo, Y., Kanie, K., Kato, T., \&. Nakamura, E. (2002). Stacking of Conical Molecules with a Fullerene Apex into Polar Columns in Crystals and Liquid Crystals, Nature, 419, 702-705.

[9] Chuard, T., Dardel, B., Deschenaux, R., \& Even, M. (2000). [60] fullerenecontaining Thermotropic Liquid Crystals, Carbon, 38, 1573-1576. 


\section{MESOGEN-DECORATED NANOPARTICLES: A MC STUDY}

[10] Cseh, L., \& Mehl, G. H. (2006). The Design and Investigation of Room

Temperature Thermotropic Nematic Gold Nanoparticles, J. Am. Chem. Soc., $128,13376-13377$.

[11] Cseh, L., \& Mehl, G. H. (2007). Structure-property Relationships in Nematic Gold Nanoparticles, J. Mat. Chem., 17, 311-315.

[12] Mang, X. B., Zeng, X. B., Tang, B. J., Liu, F., Ungar, G., Zhang, R. B., Cseh, L., \& Mehl, G. H. (2012). Control of Anisotropic Self-assembly of Gold Nanoparticles Coated with Mesogens, J. Mat. Chem., 22, 11101-11106.

[13] Wojcik, M. M., Gora, M., Mieczkowski, J., Romiszewski, J., Gorecka, E., \& Pociecha, D. (2011). Temperature-controlled Liquid Crystalline Polymorphism of Gold Nanoparticles, Soft Matter, 7, 10561-10564.

[14] Wojcik, M., Kolpaczynska, M., Pociecha, D., Mieczkowski, J., \& Gorecka, E. (2010). Multidimensional Structures Made by Gold Nanoparticles with Shape-adaptive Grafting Layers, Soft Matter, 6, 5397-5400.

[15] Wojcik, M., Lewandowski, W., Matraszek, J., Mieczkowski, J., Borysiuk, J., Pociecha, D., \& Gorecka, E. (2009). Liquid-crystalline Phases Made of Gold Nanoparticles, Angew. Chem. Int. Ed., 48, 5167.

[16] Pasini, P., Chiccoli, C., \& Zannoni, C. (2001). Liquid Crystal Lattice Models. I. Bulk systems. in Advances in the Computer Simulations of Liquid Crystals, edited by P. Pasini , \& C. Zannoni (Kluwer, Dordrecht, 2000), 545, 99. [17] Zannoni, C. (2001). Molecular Design and Computer Simulations of Novel Mesophases, J. Mat. Chem., 11, 2637-2646.

[18] Tiberio, G., Muccioli, L., Berardi, R., \& Zannoni, C. (2009). Towards in Silico Liquid Crystals. Realistic Transition Temperatures and Physical Properties for n-cyanobiphenyls via Molecular Dynamics Simulations, ChemPhysChem, 10, 125-136.

[19] Ilnytskyi, J. M., Neher, D., Saphiannikova, M., Wilson, M. R., \& Stimson, L. M. (2008). Molecular Dynamics Simulations of Various Branched Polymeric Liquid Crystals, Mol. Cryst. Liq. Cryst., 496, 186. 


\section{MESOGEN-DECORATED NANOPARTICLES: A MC STUDY}

[20] Ilnytskyi, J. M., Lintuvuori , J. S., \& Wilson, M. R. (2010). Simulation of Bulk Phases Formed by Polyphilic Liquid Crystal Dendrimers, Cond. Matter Phys., 13, 33001-3301.16.

[21] Sazonovas, A., Orlandi, S., Ricci, M., Zannoni, C., \& Gorecka, E. (2006). A Computer Simulation Study of the Ordered Phases of Some Mesogenic Fullerene Derivatives, Chem. Phys. Lett., 430, 297-302.

[22] Ellison, L. J., Michel, D. J., Barmes, F., \& Cleaver, D. J. (2006). Entropydriven Formation of the Gyroid Cubic Phase, Phys. Rev. Lett., 97, 237801.

[23] Bates, M. A., \& Walker, M. (2011). Computer Simulation of the Columnar Phases of Liquid Crystalline Bolaamphiphiles, Liq. Cryst., 38, 1749-1757.

[24] Nealon, G. L., Greget, R., Dominguez, C., Nagy, Z. T., Guillon, D., Gallani, J. L. et al. (2012). Liquid-crystalline Nanoparticles: Hybrid Design and Mesophase Structures, Beilstein J. Org. Chem., 8, 349-370.

[25] Qi, H., \& Hegmann, T. (2008). Impact of Nanoscale Particles and Carbon Nanotubes on Current and Future Generations of Liquid Crystal Displays, $J$. Mat. Chem., 18, 3288-3294.

[26] Lagerwall, J., \& Scalia, G. (2012). A New Era for Liquid Crystal Research: Applications of Liquid Crystals in Soft Matter Nano-, Bio- and Microtechnology, Curr. Appl. Phys., 12, 1387-1412.

[27] Dintinger, J., Tang, B. J., Zeng, X., Kienzler, T., Mehl, G. H., Ungar, G., et al. (2012). Optical Properties of Mesogen-coated Gold Nanoparticles, Proc. SPIE, 8271, IX 827106.

[28] Orlandi, S., Muccioli, L., Ricci, M., Zannoni, C. (2009). Self assembled fullerene walls in di-mesogenic-C60 materials, Soft Matter, 5, 4484-4491.

[29] Gay, J. G., \& Berne, B. J. (1981). Modification of the Overlap Potential to Mimic a Linear Site-site Potential, J. Chem. Phys.,74, 3316-3319. [30] Berardi, R., Emerson, A. P. J., \& Zannoni, C. (1993). Monte Carlo Investigations of a Gay-Berne Liquid Crystal, J. Chem. Soc. Faraday Trans., 89, 4069-4078. 
[ 31] Berardi, R., Fava C., \& Zannoni, C. (1998). A Gay-Berne potential for dissimilar biaxial particles. Chem. Phys. Lett., 89, 8-14.

[32] Berardi, R., Micheletti, D., Muccioli, L., Ricci, M., \& Zannoni, C. (2004). A Computer Simulation Study of the Influence of a Liquid Crystal Medium on Polymerization, J. Chem. Phys., 121, 9123-9130. 